\title{
Coronavirus Disease 2019: A Wake-Up Call for Neurosurgeons to Learn Percutaneous Tracheostomy
}

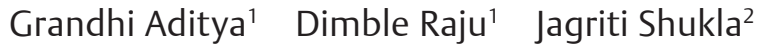 \\ ${ }^{1}$ Department of Neurosurgery, National Neurosciences Centre, \\ Calcutta, West Bengal, India \\ ${ }^{2}$ Department of Neuroanesthesiology, National Neurosciences \\ Centre, Calcutta, West Bengal, India
}

\begin{abstract}
Address for correspondence Prasad Krishnan, MS, MCh, National Neurosciences Centre, Peerless Hospital Campus, 2nd Floor, 360 Panchasayar, Kolkata-700094, West Bengal, India (e-mail: prasad.krishnan@rediffmail.com).
\end{abstract}

J Neurosci Rural Pract:2021;12:197-199
Abstract
Keywords
- aerosol
- coronavirus disease 2019
- head injury
- intubation
- open surgical tracheostomy
- percutaneous tracheostomy
- ventilator

Tracheostomy is a commonly performed operation in neurosurgical patients. It is an aerosol generating procedure and is considered a high-risk operation in times of the coronavirus disease 2019 pandemic. Though percutaneous tracheostomy has been around for some time, many neurosurgeons still perform open surgical tracheostomy as they have been trained in doing so and are well versed with the procedure. However, this pandemic is a wake-up call for them to learn a new skill that is simple, quick, and has several advantages over the traditional method.

\section{Introduction}

Tracheostomy is a common bedside procedure in neurosurgery. Patients with poor Glasgow Coma Scale and impaired cough reflex are frequently intubated and ventilated in neurosurgical intensive care units. Prolonged endotracheal intubation and ventilation is associated with complications like ventilator-associated pneumonia, vocal cord damage, difficulty in weaning, and sinusitis. ${ }^{1}$ For patients in whom airway protection is required even after the first few days, tracheostomy helps by preventing iatrogenic vocal cord injury, aids in weaning patients off the ventilator by decreasing dead space, and enabling them to breathe with a lesser tidal volume and helps in tracheobronchial toileting and treating associated respiratory tract infections. Further, it is associated with greater patient comfort and decreased sedation requirements. ${ }^{2}$
Two common techniques are in vogue: open surgical tracheostomy (OST) and percutaneous dilatational tracheostomy. ${ }^{3}$ While there are no significant differences in complication rates among the two procedures, ${ }^{3,4}$ the former has the advantage of being cheaper (no requirement of a separate set) and is learnt by residents in their first year of training. However, it has the drawback of being an aerosol generating procedure as the patient coughs, while the trachea is being incised and is risky both to the surgeon and the surrounding personnel in the setting of the current COVID-19 (novel coronavirus disease 2019) pandemic.5,6

Several studies have stated safety in OST in patients with COVID-19 can be increased by techniques like (1) advancing the endotracheal tube till the carina to prevent inadvertent rupture of the cuff while incising the trachea, ${ }^{5,7}$ (2) performing tracheostomy under muscle relaxants to prevent coughing
DOI https://doi.org/ 10.1055/s-0040-1716808 ISSN 0976-3147. (c) 2020. Association for Helping Neurosurgical Sick People.

This is an open access article published by Thieme under the terms of the Creative Commons Attribution-NonDerivative-NonCommercial-License, permitting copying and reproduction so long as the original work is given appropriate credit. Contents may not be used for commercial purposes, or adapted, remixed, transformed or built upon. (https://creativecommons.org/licenses/by-nc-nd/4.0/)

Thieme Medical and Scientific Publishers Pvt. Ltd. A-12, 2nd Floor, Sector 2, Noida-201301 UP, India 
during the procedure, ${ }^{5}(3)$ applying sutures from inferior margin of skin till the trachea to prevent false passage of the tube and to draw the trachea upwards for ease of insertion, ${ }^{7}$ and (4) switching of the ventilator during insertion to prevent movement of air in the patients respiratory tract. ${ }^{5,6}$

In the scenario of the current pandemic, we feel percutaneous tracheostomy (PCT) has an advantage over OST as (1) there is no need for any coagulation (potential for virus dissemination by cautery plumes is less), (2) lesser personnel required (no need for retraction of muscles by an assistant and a single operator can do the job even without a scrub nurse), (3) the dilatation of the tracheal opening is done in a closed manner by a dilator (passed over a guide wire inserted through a cannula via a stab incision on the neck) with no exposure of the trachea to the outside environment, (4) no fear of false passage of the tube in the pretracheal space after trachea is opened obviating the need for repeated manipulation, and finally, (5) lesser time consumed in the procedure.

We have performed 11 tracheostomies since the outbreak of the pandemic (two in COVID-19 positive patients, two in patients whose COVID-19 status was indeterminate, and seven who were COVID-19 negative) by this technique (-Figs. 1 and 2). Except in one patient where there was open depressed skull fracture with concomitant gross faciomaxillary injury (maxillofacial repair and decompressive craniotomy were done at the same sitting on the day of admission), we had deferred tracheostomy for at least 10 days from intubation to see if the patients could be safely extubated and performed it only if that was not deemed possible. This is to allow viral load in the patients' upper airway to decrease to lessen chances of the spread of infection. ${ }^{6}$ In borderline cases, no "trial of extubation" (even if COVID negative) was done if there was a risk that he might require emergency reintubation and generate aerosol-a concept proposed by other studies as well. ${ }^{6}$ All the cases were operated by a single surgeon and anesthesiologist wearing personal protective equipment (with N-95 masks, goggles, and visor) in a dedicated COVID operating room with no additional staff. All cases were operated under muscle relaxant (atracurium $0.5 \mathrm{mg} / \mathrm{kg}$ bolus dose) that would provide relaxation of 20 to 30 minutes and prevent "bucking" during the procedure. The ventilator was switched off to produce transient apnea for 30 to 40 seconds during the time of dilation of the tract and insertion of the tracheostomy tube. Position was confirmed by seeing end tidal $\mathrm{CO}_{2}$ tracing on the monitor after removing the obturator.

We believe that PCT is an easy, safe and fast procedure which properly performed will generate fewer aerosols than OST and the current pandemic should arouse neurosurgeons to learn this technique quickly.

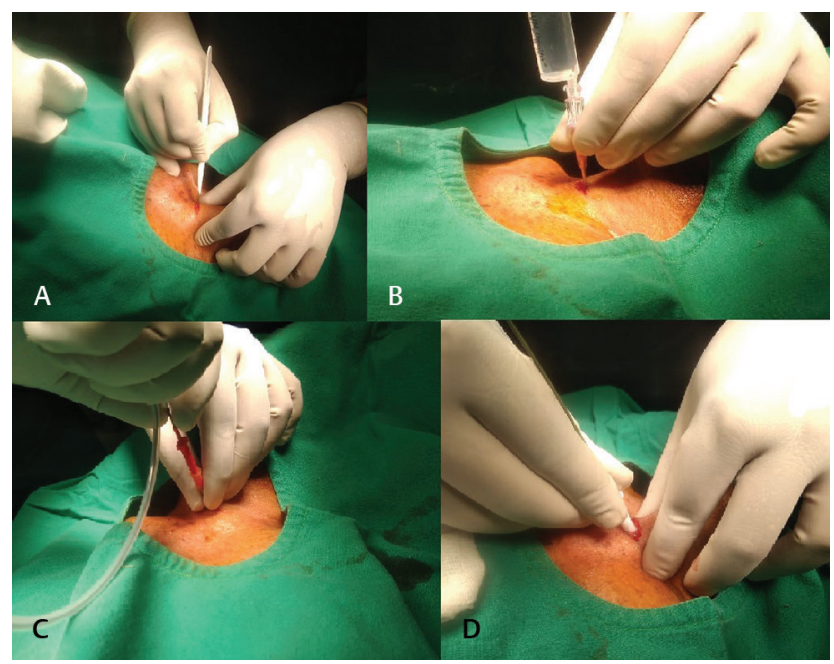

Fig. 1 (A) A stab incision being made on the skin, (B) a bevelled needle with a cannula passed into the trachea and air aspirated to confirm its correct location, (C) guide wire being passed through the cannula, and (D) after removing the cannula a predilator used to open up the trachea over the guide wire holding the trachea to prevent its displacement.

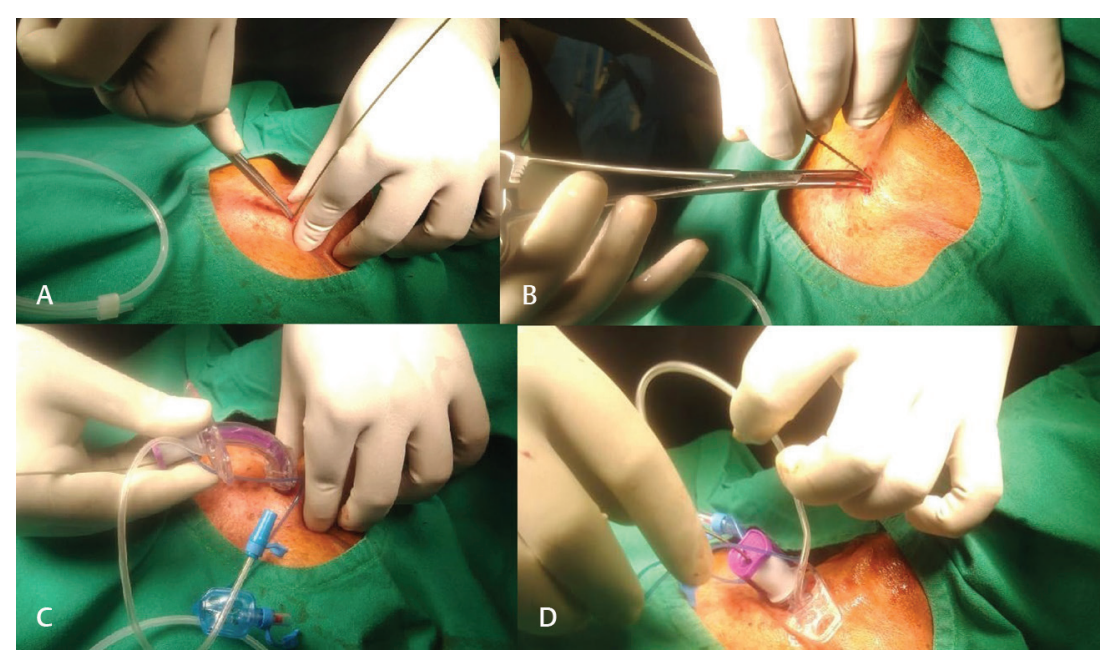

Fig. 2 (A) A cannulated dilator being used to open subcutaneous spaces and (B) dilate trachea, (C) the tracheostomy tube being passed over the guide wire once the tract is dilated, and (D) the cuffed tube finally in position prior to removal of the obturator. 


\section{Funding}

None.

\section{Conflict of Interest}

None declared.

\section{References}

1 Quiñones-Ossa GA, Durango-Espinosa YA, Padilla-Zambrano H, et al. Current status of indications, timing, management, complications, and outcomes of tracheostomy in traumatic brain injury patients. J Neurosci Rural Pract 2020;11(2):222-229

2 Freeman BD. Tracheostomy update: when and how. Crit Care Clin 2017;33(2):311-322

3 Klotz R, Probst P, Deininger M, et al. Percutaneous versus surgical strategy for tracheostomy: a systematic review and meta-analysis of perioperative and postoperative complications. Langenbecks Arch Surg 2018;403(2):137-149

4 Johnson-Obaseki S, Veljkovic A, Javidnia H. Complication rates of open surgical versus percutaneous tracheostomy in critically ill patients. Laryngoscope 2016;126(11):2459-2467

5 Zhang X, Huang Q, Niu X, et al. Safe and effective management of tracheostomy in COVID-19 patients. Head Neck 2020; 42(7):1374-1381

6 McGrath BA, Brenner MJ, Warrillow SJ, et al. Tracheostomy in the COVID-19 era: global and multidisciplinary guidance. Lancet Respir Med 2020;8(7):717-725

7 Mattioli F, Fermi M, Ghirelli M, et al. Tracheostomy in the COVID-19 pandemic. Eur Arch Otorhinolaryngol 2020;277(7): 2133-2135 